\title{
Use of Modern Contraceptive Methods among Migrant Women Living in Slovenia
}

\section{Igor Karnjuš}

University of Primorska, Slovenia

igor.karnjus@fvz.upr.si

\section{Mirko Prosen}

University of Primorska, Slovenia

mirko.prosen@fvz.upr.si

Urška Bogataj

University of Primorska, Slovenia urska.bogataj@fvz.upr.si

\section{Doroteja Rebec}

University of Primorska, Slovenia

doroteja.rebec@fvz.upr.si

\section{Sabina Ličen}

University of Primorska, Slovenia

sabina.licen@fvz.upr.si

The use of modern contraceptives by immigrants may be constrained by various factors such as poor language skills, socio-economic background, and inadequate knowledge. Limited evidence is available on the factors associated with the use of modern contraceptive methods by migrant women in Slovenia. The study included 52 migrants who were treated in a maternity hospital in Slovenia between March and September 2018. Data were collected using a questionnaire that contained items related to sexual and reproductive health. Bivariate analysis (Spearman's correlation coefficient and Chi square) was used to examine the association between independent variables and the use of contraceptive methods. The study highlights that two main factors affect contraception use: (a) Migrant women with better knowledge of modern contraceptive methods were more likely to use them ( $r=0.69 ; p<0.05)$; (b) If the partner/husband has a positive attitude to the use of modern contraceptives, there is a greater probability migrant women will use them $\left(\chi^{2}=14.465\right.$; $p=0.001$ ). Based on the results, sexual and reproductive health education for migrant women and men, particularly on the use of modern contraception, is needed.

\section{Introduction}

Migration has always been a major cause of population change. People migrate from low-income countries to high-income ones for better jobs and 
to improve their socio-economic circumstances. Further, in recent years the presence of wars around the world has also been affecting migration flows (Adanu \& Johnson, 2009). The European Union (EU) is today facing the most serious migration crisis since World War II (European Migration Network, 2017). Despite the general belief that the lion's share of migrants is men, since migration is generally associated with the search for work, data for 2015 show that women made up about $48.0 \%$ of international migrants worldwide (Shah, Kiriya, Shibanuna, \& Jimba, 2018). Migration is a major event in life that can have an extreme impact on women's sexual and reproductive health (SRH). In fact, studies demonstrate that migrant women's SRH can be negatively affected by the challenges of migration (Botfield, Newman, \& Zwi, 2016; McMichael \& Gifford, 2010). Moreover, depending on the policies and practices of the host country regarding migrants, they may experience discrimination and a loss of their socio-economic status (Mengesha, Perz, Dune, \& Ussher, 2017).

$\mathrm{SRH}$ is one of the most important components of quality of life (Metusela et al., 2017) which includes 'physical, emotional, mental and social well-being' as well as 'pleasurable, safe sexual experiences that are free from coercion, discrimination or violence' (WHO, 2010). The use of SRH care is associated with improved mental health and nutrition, positive economic and social outcomes for women, as well as clinical benefits such as reduced rates of unplanned pregnancy and sexually transmitted infection (Cohen, 2004; Mengesha et al., 2017).

However, the literature states (Keygnaert et al., 2014) that migrant women are, compared to the EU population, less often screened for cervical and breast cancer, made fewer visits to a gynaecologist, have more induced abortions and pregnancy complications, and have less access to family planning and contraception. Further, migrant women are at greater risk of sexually transmitted infections. Although SRH is an important aspect of women's quality of life, migrant women have much less access to SRH services than the EU population (Sebo, Jackson, Haller, Gaspoz, \& Wolff, 2011). At the beginning, due to resettlement changes, migrant women attribute low importance to sexual health needs, and knowledge about health resources and services in the host country is often very limited (Metusela et al., 2017). While knowledge is a major barrier regarding SRH behaviour, the latter may be influenced by various factors such as cultural and religious norms from the country of origin. Migrant women often come from countries in which they hold a disadvantaged position in society. These norms affect the acquisition of sexual health literacy and behaviours, and can contribute to a lack 
of knowledge and access to SRH services (Metusela et al., 2017; Raben \& Mujisenbergh, 2018). Among some migrant communities, discussing a person's sexual needs is often forbidden since it is still considered a taboo. In such cases, sexual healthcare services may be seen as culturally inappropriate, and are thus avoided (Guerin, Allotey, Hussein Elmi, \& Baho, 2006; Rogers \& Earnest, 2015). Consequently, many migrant women may possess little knowledge about risk factors for unwanted pregnancies and about contraception due to limited health literacy. Young migrant adults often do not receive sufficient education on sexual health, and unmet contraceptive needs are common (Aptekman, Rashid, Wright, \& Dunn, 2014; Raben et al., 2018).

Based on effectiveness level, contraceptive methods can be categorised in three large groups. ${ }^{1}$ The safest contraception methods include male and female sterilisation, implants, and intrauterine devices. Pills, injectables, vaginal rings, hormonal patches, and the diaphragm are placed in the group of mid-level effectiveness. The least effective methods of contraception are considered male and female condoms, spermicides, withdrawal, and fertilityawareness-based methods. However, migrant women may be unaware of the newest modern contraception methods available in the host country. In addition, women from less developed countries are less likely to use them, which probably influences their lower use even after migration (Shah et al., 2018). In fact, the use of contraception is affected by access to affordable and preferred contraception, and to supportive SRH services. For immigrants, access is made even more difficult by social and religious differences and linguistic barriers (Ellawela et al., 2017). It should be emphasised that migrant women may improve their quality of life by using modern contraceptive methods. They can reduce the fear of unwanted pregnancy, which is present when using traditional methods such as withdrawal and the rhythm method. Consequently, this can have a positive impact on sexual life and promote psychosocial well-being (Caruso et al., 2016). Hormonal contraceptives also provide medical benefits such as a decrease in menstrual pain and regulation of the menstrual cycle (Shah et al., 2018).

Yet, contraceptive awareness and their use among migrant women is poorly explored in the academic literature, even though it is a key determinant of SRH. Limited evidence is available on the factors associated with the use of contraceptive methods by migrant women. Thus, the study examines the factors associated with the modern contraceptive methods used by migrant women in Slovenia.

\footnotetext{
${ }^{1}$ See https://www.cdc.gov/reproductivehealth/contraception/index.htm.
} 


\section{Methods}

\section{Study Design}

A quantitative, cross sectional design was employed. The study is part of the INTEGRA project (INTERREG programme V A Italy-Slovenia 2014-2020) concerning migrant women's population characteristics, as well as their sexual and reproductive health and social integration into Italy and Slovenia.

\section{Sample}

Convenience sampling was used. Migrant women treated in a maternity hospital in Slovenia between March and September 2018 as either an ambulatory or hospitalised patient were invited to participate in the study. Participation in the study was voluntary. Only adult migrant women older than 18 years were included in the study.

\section{Instrument}

The research instrument was a semi-structured questionnaire developed by the lead partner (University of Trieste in association with the Burlo Garofolo Pediatric Institute) and submitted in January 2018 (16.1.2018; 12:11). The Italian version of the questionnaire was translated into the Slovenian language and adapted to the Slovenian cultural context, and aligned with the healthcare system established in the Republic of Slovenia. Finally, the questionnaire was reviewed by a panel of experts composed of five independent nursing lecturers. Besides the socio-demographic part, the questionnaire contains 69 items related to the social integration of migrant women in the host county and their sexual and reproductive health.

\section{Data Collection Procedure}

The survey was conducted between March and September 2018. The questionnaire was distributed with the assistance of hospital staff involved in the project. Two midwives were additionally provided with a one-day training session to familiarise them with the study's objectives, the study protocol and the questionnaire contents. They also supported migrant women while responding to the questionnaire if needed (e.g. linguistic barriers).

The participants' confidentiality and anonymity were assured and maintained during the completion and submission of the questionnaire. Oral informed consent was obtained. All participants were informed about the aims, objectives and study methods used. The study was conducted in accordance with the Helsinki-Tokyo Declaration and the Code of Ethics for Nurses and 
Nurse Assistants of Slovenia. The study was approved by the National Medical Ethics Committee (26.10.2017; No. 0120-544/2017/7).

\section{Data Analysis}

Data were processed and analysed using SPSS version 23 (SPSS Inc., Chicago, IL, USA). Bivariate analysis (Spearman's correlation coefficient and Chi square) was conducted to examine the association between the independent variables and the use of contraceptive methods. Statistical significance was set at $p<0.05$.

\section{Results}

\section{Demographic Data of the Sample}

The convenience sample included 52 migrant women. The participants' average age was $32.46(s=8.06)$. The youngest was 19 and the oldest 56 years. The survey included migrant women who left their country of birth between 2003 and 2018. Most participants ( $n=32 ; 61.5 \%$ ) had migrated to Slovenia in the last 5 years (2014-2018). The length of their stay in Slovenia varied from less than 1 year to 15 years (mean $=4.16$ years, $s=7.25$ ). The participants' average age when arriving in Slovenia was 27.53 ( $s=7.25$ ) years; the youngest participant was 17 and the oldest 53 years.

Table 1 summarises some of the socio-demographic characteristics of the migrant women participating in the study. Most of these women come from the former republics of Yugoslavia $(n=36 ; 69.2 \%)$ or Russia $(n=11 ; 21.2 \%)$. Half the participants claimed they are members of the Orthodox Church $(n=26$; $50.0 \%)$, followed by members of Islam $(n=19 ; 36.5 \%)$. Regarding their current employment status in Slovenia, 28 migrant women (53.8\%) stated they are unemployed or run a household and are at home; 24 (46.1\%) women stated they have a job. The majority of these women $(n=34 ; 65.4 \%)$ had lived in a city in their country of birth; 18 (34.6\%) stated they had lived in the countryside. The majority of the migrant woman $(n=39 ; 75.0 \%)$ stated they already had one or more children, while 13 (25.0\%) had no children. Ten migrant women $(19.2 \%)$ included in the study had one or more induced abortions in the past.

\section{Knowledge and Current Use of Contraceptive Methods}

Half the participants $(n=26 ; 50.0 \%)$ stated that upon arrival in Slovenia they were given access to new methods of contraception. Tables 2 and 3 illustrate the use of contraceptive methods and knowledge concerning them among the migrant women participating in the study. In answering these two questions, participants were able to give multiple responses. The modern contra- 
Table 1 Socio-Demographic Characteristics of The Migrant Women

\begin{tabular}{llrr}
\hline Variables & & $n$ & $\%$ \\
\hline Country of birth & Bosnia and Herzegovina & 16 & 30.8 \\
& Croatia & 2 & 3.8 \\
& Kosovo & 5 & 9.6 \\
& FYR Macedonia & 9 & 17.3 \\
& Russia & 11 & 21.2 \\
& Slovak Republic & 1 & 1.9 \\
& Serbia & 4 & 7.7 \\
& Ukraine & 4 & 7.7 \\
\hline Education & $<$ Higher secondary & 10 & 19.2 \\
& Higher secondary & 20 & 38.5 \\
& $\geq$ Undergraduate & 22 & 42.3 \\
\hline Religion & Catholic & 2 & 3.8 \\
& Protestant & 2 & 3.8 \\
& Muslim & 19 & 36.5 \\
& Orthodox & 26 & 50.0 \\
& Other & 1 & 1.9 \\
& Not a member of any religion & 2 & 3.8 \\
\hline No. of children & Housewife & 17 & 32.7 \\
\hline Employment status & Unemployed & 11 & 21.2 \\
& Employed & 24 & 46.1 \\
\hline & None & 13 & 25.0 \\
& 1 & 13 & 25.0 \\
& $\geq 2$ & 50.0 \\
\hline
\end{tabular}

ception methods migrant women mostly use are oral contraceptive pills and condoms. While only a small number of participants claimed they also use traditional methods, this was always in combination with some other modern contraceptive method. However, 21 (40.4\%) participants stated they did not use any contraception method. Regarding their knowledge of modern contraceptives, of the 43 participants who answered this question, $37 \mathrm{knew}$ of at least one modern contraceptive method (with the number ranging from 1 to 7). Migrant women are least familiar with the use of subcutaneous implants, patches and hormonal injections.

The participants were also asked whether their partner agrees to the use of contraception. Eleven migrant women (21.6\%) stated their partner disagrees with the use of contraception; 4 (7.7\%) participants stated he is indifferent on this question and $32(61.5 \%)$ stated that he agrees with its use. For a ques- 
Table 2 Current Use of Contraceptive Methods among the Migrant Women

\begin{tabular}{llrr}
\hline Contraceptive methods $(n=52)$ & & $n$ & $\%$ \\
\hline Modern methods & Oral contraceptive pills & 14 & 26.9 \\
& Condom & 20 & 38.5 \\
& Diaphragm & 1 & 1.9 \\
& Intrauterine device & 3 & 5.8 \\
& Subcutaneous implants & 0 & 0.0 \\
& Patches & 1 & 1.9 \\
& Hormonal injections & 3 & 5.8 \\
\hline Traditional methods & Rhythm method & 3 & 5.8 \\
& Withdrawal & 3 & 5.8 \\
\hline Others & & 0 & 0.0 \\
\hline None & & 21 & 40.4 \\
\hline
\end{tabular}

Notes Multiple responses allowed.

Table 3 Knowledge of Contraceptive Methods among the Migrant Women

\begin{tabular}{llrr}
\hline Contraceptive methods $(n=43)$ & & $n$ & $\%$ \\
\hline Modern methods & Oral contraceptive pills & 36 & 83.7 \\
& Condom & 36 & 83.7 \\
& Diaphragm & 20 & 46.5 \\
& Intrauterine device & 21 & 48.8 \\
& Subcutaneous implants & 11 & 25.6 \\
& Patches & 15 & 34.8 \\
& Hormonal injections & 15 & 34.8 \\
\hline
\end{tabular}

Notes Multiple responses allowed.

tion regarding the type of contraception used by women, 41 (78.8\%) participants stated this is exclusively the decision of the woman, 4 (7.7\%) participants stated this is a joint decision of the two partners and 4 (7.7.\%) stated that it is a decision of the husband/partner.

\section{Factors Associated with the Use of Contraceptive Methods}

Table 4 presents an analysis of factors associated with the migrant women's use of contraceptive methods. Two main factors influencing the use of contraception are knowledge regarding modern contraceptive methods and the partner's attitude to their use. In fact, migrant women with better knowledge of modern contraceptive methods were more likely to use them. Further, if the partner has a positive attitude to contraception use there is a greater probability the woman will use it. We were unable to determine the influ- 
Table 4 Factors Associated with the Currently Used Contraceptive Methods

\begin{tabular}{llrrrr}
\hline Variables & & Yes & No & $p$-value & \\
\hline Living environment & City & 23 & 11 & 0.140 & $\left(\chi^{2}=2.632\right)$ \\
in country of birth & Countryside & 8 & 10 & & \\
\hline Education & < Higher secondary & 3 & 7 & 0.103 & $\left(\chi^{2}=4.554\right)$ \\
& Higher secondary & 13 & 7 & & \\
& $\geq$ Undergraduate degree & 15 & 7 & & \\
\hline Partner's attitude & Disagrees & 2 & 9 & $0.001^{* *}\left(\chi^{2}=14.465\right)$ \\
to contraception & Neutral & 2 & 2 & & \\
& Agrees & 26 & 6 & & \\
\hline History of abortion & Yes & 8 & 2 & 0.280 & $\left(\chi^{2}=1.927\right)$ \\
& No & 23 & 18 & & \\
\hline No. of children & None & 10 & 3 & 0.382 & $\left(\chi^{2}=1.923\right)$ \\
& 1 & 7 & 6 & & \\
\hline Contraceptive knowledge & 14 & 11 & & \\
\hline Age & $\geq 2$ & \multicolumn{5}{c}{$0.031^{*}$} & $(r=0.690)$ \\
\hline Length of stay in host country & & & & 0.243 & $(r=0.165)$ \\
\hline
\end{tabular}

Notes * Significant at the level $p<0.05 .{ }^{*}$ Significant at the level $p<0.001$.

ence of other factors like length of stay in the country, education level etc., on use of contraceptive methods (Table 3 ).

We also analysed the influence of religion and country of birth on the use of contraceptive methods. Although we found no statistically significant differences between the subgroups (religion: $\chi^{2}=4.166, p=0.526$; country of birth: $\chi^{2}=8.403, p=0.298$ ), women from Bosnia and Herzegovina, FYR Macedonia and Kosovo use contraception somewhat less than women from other countries. Of 30 women from these countries, 14 do not use any contraception.

\section{Discussion}

The key findings of this study are: (1) Migrant women who possessed better knowledge of modern contraceptive methods were more likely to use them; (2) If the partner/husband has a positive attitude to the modern contraceptive use, there is a greater probability a migrant woman will use them.

Several studies show that knowledge of contraception has a great influence on its use in everyday life, not only among immigrants. There is an established link between knowledge and responsible sexually protective behaviours (Cleland, Conde-Agudelo, Peterson, Ross, \& Tsui, 2012; Poncet, 
Huang, Rei, Lin, \& Chen, 2013; Raben et al., 2018; Shah et al., 2018). In our study, 37 migrant women (71.2\%) claimed they are familiar with at least one contraception method among those listed, and 11 stated they are familiar with all 7 modern methods on the list. The least familiarity was found with newer methods like subcutaneous implants, patches and hormonal injections. Several authors emphasise that among migrant women knowledge regarding contraception is mainly sourced from peers, the media, magazines and other informal sources allowing incorrect knowledge and myths to be present among community members (Metusela et al., 2017; Ip et al., 2011). Therefore, the danger of passing false information on regarding contraception among this population should be taken seriously.

In recent years, the range of contraceptives available has expanded and the overall distribution of contraceptive methods has changed. The use of permanent methods such as sterilisation has decreased, and barrier and long-acting hormonal methods have increased (Ellawela et al., 2017). In our study, $50.0 \%$ of the migrant woman stated that upon arriving in Slovenia they were given access to new contraception methods. The most common form of contraception reported by the participants is the use of oral contraceptive pills and condoms. Women's choice of contraceptive methods is affected by demographics, socio-economic status, cultural norms, level of commitment to avoiding pregnancy, and perceived access to healthcare (Poncet et al., 2013). Further, Raben et al. (2018) emphasise that migrant women tend to adapt to the contraceptive trends in their host countries. According to the National Institute of Public Health, hormonal contraception is currently the most commonly used contraceptive method in Slovenia (see http://www.nijz.si/sl/26-september-2014-svetovni-dan-kontracepcije). A report from 2016 states that almost $94.0 \%$ of hormonal contraception is due to the use of oral contraceptive pills. The latter are mostly used by young women between the ages of 15 and 30. Young woman at the start of a new sexual relationship, in an unstable relationship or having sex with multiple partners most commonly use condoms as a contraception method. Intrauterine devices are the typical birth control method used by women aged between 30 and 44. In Slovenia, the use of permanent methods like sterilisation is very low; estimated at around $3.0 \%$ of couples.

Another important factor influencing the use of contraception among couples is the partner's attitude to its use. Women are more likely to use contraception when their partner/husband agrees with the decision and when members of their community approve of family planning (Poncet et al., 2013), which was confirmed by our results. A study by Zheng et al. (2001) demon- 
strated that inequality between partners may affect the woman's contraceptive attitude since young women were found to rely on their partners when making decisions on contraceptive use. Culturally prescribed gender roles, combined with patriarchal values, may affect a woman's ability to have control over her sexual and reproductive needs (Metusela et al., 2017). However, it has been reported that in some cases migration to an economically more developed country can result in positive changes in men's attitudes to sexual and reproductive health (Adanu \& Johnson, 2009). The use of contraceptives can be further enhanced by good communication between partners. A study by Kamal and Islam (2012) showed that frequent communication between partners about family planning leads to greater use of contraceptives.

Level of education is also recognised as an important factor in family planning. Although we were unable to demonstrate the influence of this factor on contraception use, the utilisation of modern contraceptives in this study was somewhat higher among immigrants who possessed a higher education. A French survey investigating the use and method of contraception among immigrant and non-immigrant women found that immigrant women with a lower level of educational attainment were less likely to use contraception (Poncet et al., 2013). Similar results were found in previous studies in China and Australia (Ip, Chan, Chan, \& Chan, 2011; Shah et al., 2018). Women with a higher level of education have a better social status, which gives them more options for contraception and they are thus more likely to use them (Shaukat \& Pell, 2015).

We also observed that migrant women from some former republics of $\mathrm{Yu}$ goslavia which are less economically developed, like Bosnia and Herzegovina, FYR Macedonia and Kosovo, tend to use contraception less than women from other countries. The lower utilisation rate of contraceptives may be partly the result of complex social processes associated with an immigration background, such as values and norms involving contraceptive practices, socio-cultural beliefs, awareness or knowledge of reproductive health or the freedom to make safe reproductive choices (Poncet et al., 2013). Although people from the former Yugoslav republics (mainly Bosnia and Herzegovina, Macedonia, Serbia and Kosovo) still account for most of the foreign immigrants in Slovenia, the country has increasingly become a target country for other immigrants like Iranians, Syrians, people from Afghanistan etc. (European Migration Network, 2017). We must be aware that today and in the near future the number of immigrants settling in high-income countries will grow, and that it is essential to have culturally competent healthcare staff in place for that scenario because every woman, regardless of her background 
or origin, has the right to adequate SRH care (Raben et al., 2018). Interventions designed to raise awareness and use of contraceptives among the migrant population can bring multiple payoffs (Lindstrom \& Hernández, 2006). It is therefore important for health services to provide culturally relevant and readily understood information to enable migrant women and men to make informed decisions about contraception (Ellawela et al., 2017).

Several limitations of this study should be considered. The sample size was relatively small $(n=52)$. In addition, our study only included migrant women treated at just one regional maternity hospital in Slovenia. Therefore, caution needs to be taken when generalising the study's findings. In addition, the study's duration was quite limited (March to September 2018). Although our questionnaire took a broad approach to the subject of migration (it also included issues related to the social integration of migrant women and not only subjects regarding their $\mathrm{SRH}$ ), in the future it should be focused on a more specific area. Despite these limitations, this study is one of the first to identify the factors associated with contraceptive use among migrant women in Slovenia and, as such, provides a good platform for further research in this area.

\section{Conclusions}

This study showed that migrant women are more likely to use modern contraceptive methods when they have better knowledge of them and if their partner has a positive attitude to contraception use. These results suggest there is a need for SRH education courses for all migrants, both male and female, especially pertaining to modern contraceptive use. Targeted culturally appropriate campaigns to improve awareness of and access to affordable contraceptive options are recommended. This study also informs the necessity to conduct a large-scale epidemiological study on the sexual and reproductive health of migrant women in Slovenia in the near future.

\section{References}

Adanu, R. M., \& Johnson, T. R. (2009). Migration and women's health. International Journal of Gynaecology and Obstetrics, 106(2), 179-181.

Aptekman, M., Rashid, M., Wright, V., \& Dunn, S. (2014). Unmet contraceptive needs among refugees. Canadian Family Physician, 6o(12), e613-9.

Botfield, J. R., Newman, C. E., \& Zwi, A. B. (2016). Young people from culturally diverse backgrounds and their use of services for sexual and reproductive health needs: A structured scoping review. Sexual Health, 13(1), 1-9.

Caruso, S., Iraci, M., Cianci, S., Fava, V., Casella, E., \& Cianci, A. (2016). Comparative, open-label prospective study on the quality of life and sexual func- 
tion of women affected by endometriosis-associated pelvic pain on $2 \mathrm{mg}$ dienogest $/ 30 \mu \mathrm{g}$ ethinyl estradiol continuous or $21 / 7$ regimen oral contraceptive. Journal of Endocrinological Investigation, 39(8), 923-931.

Cleland, J., Conde-Agudelo, A., Peterson, H., Ross, J., \& Tsui, A. (2012). Contraception and health. Lancet, 380(9837), 149-156.

Cohen, S. A. (2004). The broad benefits of investing in sexual and reproductive health. The Guttmacher Report on Public Policy, 7(1), 5-8.

Ellawela, Y., Nilaweera, I., Holton, S., Rowe, H., Kirkman, M., Jordan, L., ... Fisher, J. (2017). Contraceptive use and contraceptive health care needs among Sri Lankan migrants living in Australia: Findings from the understanding fertility management in contemporary Australia survey. Sexual \& Reproductive Healthcare, 12, 70-75.

European Migration Network. 2017. Kratko poročilo o gibanju migrantov v Sredozemlju. Retrieved from https://emm.si/publikacije/emn-kratko -porocilo-o-gibanju-migrantov-v-sredozemlju-december-2017/

Guerin, P. B., Allotey, P., Hussein Elmi, F., \& Baho, S. (2006). Advocacy as a means to an end: Assisting refugee women to take control of their reproductive health needs. Women \& Health, 43(4), 7-25.

Ip, W. Y., Chan, M. Y., Chan, D. S., \& Chan, C. W. (2011). Knowledge of and attitude to contraception among migrant woman workers in mainland China. Journal of Clinical Nursing, 20(11-12), 1685-1695.

Kamal, S. M., \& Islam, M. A. (2012). Interspousal communication on family planning and its effect on contraceptive adoption in Bangladesh. Asia-Pacific Journal of Public Health, 24(3), 506-521.

Keygnaert, I., Guieu, A., Ooms, G., Vettenburg, N., Temmerman, M., \& Roelens, K. (2014). Sexual and reproductive health of migrants: Does the EU care? Health Policy, 114(2-3), 215-225.

Lindstrom, D. P., \& Hernández, C. H. (2006). Internal migration and contraceptive knowledge and use in Guatemala. International Family Planning Perspectives, 32(3), 146-153.

McMichael, C., \& Gifford, S. (2010). Narratives of sexual health risk and protection amongst young people from refugee backgrounds in Melbourne, Australia. Culture, Health \& Sexuality, 12(3), 263-277.

Mengesha, Z. B., Perz, J., Dune, T. \& Ussher, J. (2017) Refugee and migrant women's engagement with sexual and reproductive health care in Australia: A socio-ecological analysis of health care professional perspectives. PLoS One, 12(7), e0181421.

Metusela, C., Ussher, J., Perz, J., Hawkey, A., Morrow, M., Narchal, R., Estoesta, J. \& Monteiro, M. (2017). 'In my culture, we don't know anything about that:' Sexual and reproductive health of migrant and refugee women. International Journal of Behavioral Medicine, 24(6), 836-845.

Poncet, L. C, Huang, N., Rei, W., Lin, Y. C., \& Chen, C. Y. (2013). Contraceptive use 
and method among immigrant women in France: Relationship with socioeconomic status. The European Journal of Contraception \& Reproductive Health Care, 18(6), 468-479.

Raben, L. A. D., \& van den Muijsenbergh, M. E. T. C. (2018). Inequity in contraceptive care between refugees and other migrant women? A retrospective study in Dutch general practice. Family practice, 35(4), 468-474.

Rogers, C., \& Earnest, J. (2015). Sexual and reproductive health communication among Sudanese and Eritrean women: An exploratory study from Brisbane, Australia. Culture, Health \& Sexuality, 17(2), 223-236.

Sebo, P., Jackson, Y., Haller, D. M., Gaspoz, J. M., \& Wolff, H. (2011). Sexual and reproductive health behaviors of undocumented migrants in Geneva: A cross sectional study. Journal of Immigrant and Minority Health, 13(3), 510517.

Shah, R., Kiriya, J., Shibanuma, A., \& Jimba, M. (2018). Use of modern contraceptive methods and its association with QOL among Nepalese female migrants living in Japan. PLoS One, 13(5), e0197243.

Shaukat, S., \& Pell, A. W. (2015). Personal and social problems faced by women in higher education. FWU Journal of Social Sciences, 9(2), 101-107.

World Health Organisation. (2010). Measuring sexual health: Conceptual and practical considerations and related indicators. Geneva, Switzerland: Author.

Zheng, Z., Zhou, Y., Zheng, L., Yang, Y., Zhao, D., Lou, C., \& Zhao, S. (2001). Sexual behaviour and contraceptive use among unmarried, young women migrant workers in five cities in China. Reproductive Health Matters, 9(17), 118127.

S. Ličen, I. Karnjuš, \& M. Prosen (Eds.). (2019). Women, migrations and health: Ensuring transcultural healthcare (pp. 81-93). Koper, Slovenia: University of Primorska Press. https://doi.org/10.26493/978-961-7055-43-6.81-93 